Engineering Physics and Mathematics Division

\title{
POSITION AND FORCE CONTROL OF A VEHICLE WITH TWO OR MORIE STEERABLE DRIVE WHEELS
}

David B. Reister and Michael A. Unseren

DATE PUBLISHED - October 1992

Research sponsored by the Engineering Research Program Office of Basic Energy Sciences U.S. Department of Energy

Prepared by the

OAK RIDGE NATIONAL LABORATORY

Oak Ridge, Tennessee 37831 managed by

MARTIN MARIETTA ENERGY SYSTEMS, INC. for the U.S. DEPARTMENT OF ENERGY under contract DE-AC05-84OR21400 


\section{CONTENTS}

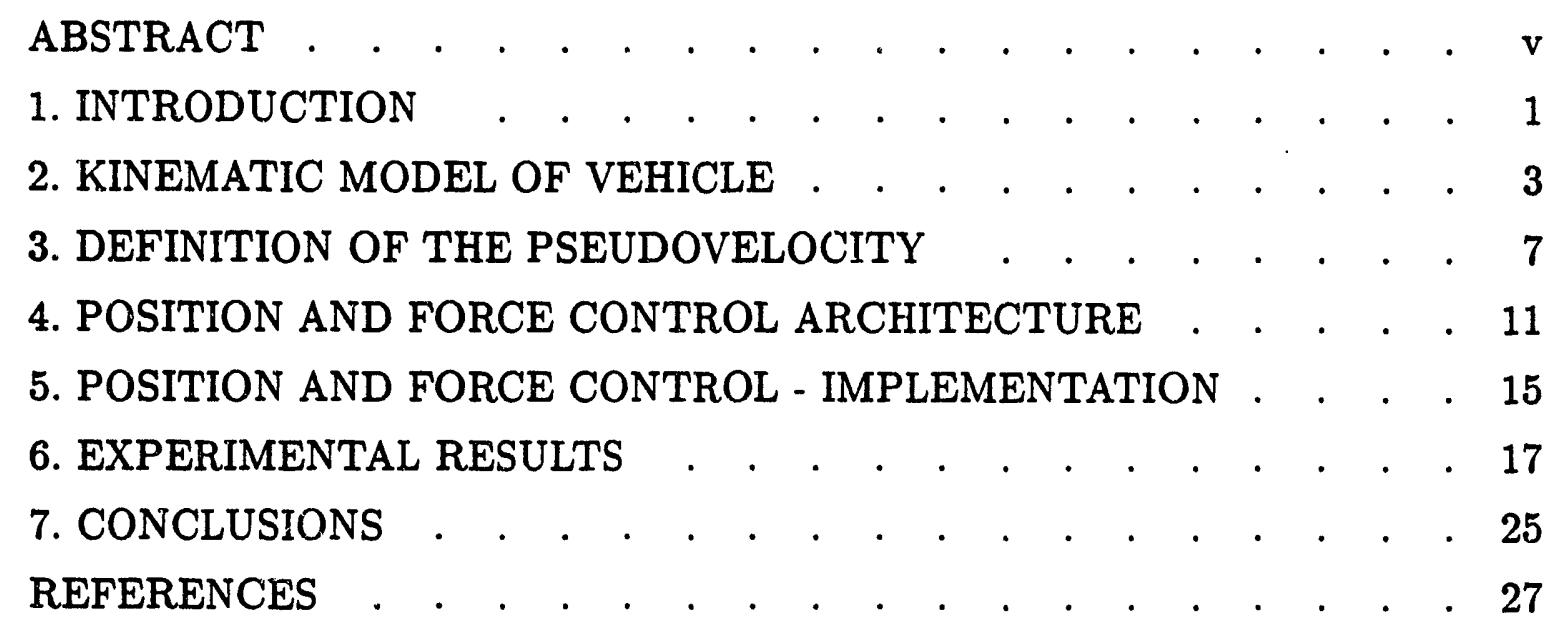




\section{LIST OF FIGURES}

Fig. $\quad$ Page

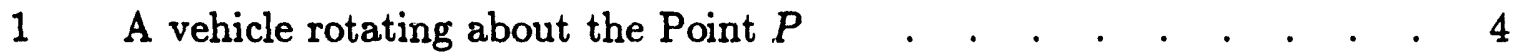

2 The rotation ratio for the first experiment (the goal is 1.2088) . . 21

3 The rotation ratio for the second experiment (the goal is 1.0) . . 22

4 The control signals $\left(u_{1}\right.$ and $\left.u_{2}\right)$ for Case 3 . . . . . . . . . . . 23

$5 \quad$ The output torque values $\left(\tau_{1}=t R\right.$ and $\left.\tau_{2}=t L\right)$ for Case 3 . . . 24 


\begin{abstract}
When a vc'.icle with two or more steerable drive wheels is traveling in a circle, the motion of the wheels is constrained. The wheel translational velocity divided by the radius to the center of rotation must be the same for all wheels. When the drive wheels are controlled independently using position control, the motion of the wheels may violate the constraints and the wheels may slip. Consequently, substantial errors can occur in the orientation of the vehicle. A vehicle with $N$ drive wheels has $(N-1)$ constraints and one degree of freedom. We have developed a new approach to the control of a vehicle with $N$ steerable drive wheels. The novel aspect of our approach is the use of force control. To control the vehicle, we have one degree of freedom for the position on the circle and $(N-1)$ forces that can be used to reduce errors. Recently, Kankaanranta and Koivo developed a control architecture that allows the force and position degrees of freedom to be decoupled. In the work of Kankaanranta and Koivo the force is an exogenous input. We have made the force endogenous by defining the force in terms of the errors in satisfying the rigid body kinematic constraints. We have applied the control architecture to the HERMIES-III robot and have measured a dramatic reduction in error (more than a factor of 20) compared to motions without force control.
\end{abstract}




\section{INTRODUCTION}

A wheel is the classic textbook example of a system that must satisfy a nonholonomic constraint. At each instant, a wheel rolling on a horizontal plane without slipping can move in only one direction. Motion in the orthogonal direction requires maneuvering. The most familiar example of the maneuvering required by a nonholonomic vehicle is parallel parking of a car. The goal is to move the car two meters in the direction orthogonal to the wheels. The optimum path is arc-line-arc and is accomplished by: turning the wheels to one limit, backing, straightening the wheels, backing, turning the wheels to the other limit, backing, and straightening the wheels (several iterations may be required by an unskillful driver).

A vehicle with steerable wheels can use the steering degrees of freedom to reduce the maneuvering. For example, a car with four steerable wheels can parallel park by turning the four wheels sideways and moving directly into the parking space. In addition to moving in a line in any direction, a vehicle with steerable wheels can move in a circle about any center of rotation. Circular motion is useful for the working around circular objects like storage drums or tanks.

Cars are becoming more like computer controlled mobile robots. Cars are available with four wheel drive, four wheel steering, and anti-lock braking systems. A team from Nippondenso and the University of California at Berkeley has developed an experimental control system for a laboratory vehicle with four steerable drive wheels. ${ }^{5}$

However, when a vehicle with two or more steerable drive wheels is traveling in a circle, the motion of the wheels is constrained. The wheel translational velocity divided by the radius to the center of rotation must be the same for all wheels. When the drive wheels are controlled independently, errors occur and the wheels will slip. Our objective is to develop a method to control constrained wheels as a unit rather than independently. The motivation for our interest in this problem was provided by the development of a wheel control system for the HERMIES-III robot. ${ }^{6}$

HERMIES-III is a large $(800 \mathrm{~kg})$ robot designed for human scale experiments. The chassis $(1.6 \mathrm{~m} \times 1.3 \mathrm{~m} \times 1.9 \mathrm{~m})$ has two steerable drive wheels and four corner caster wheels. The current wheel control system for HERMIES-III provides independent velocity control of the rotation of the two drive wheels. During constrained circular motion, substantial errors can occur. For example, the goal might be a rotation of 10 degrees about the point $(2,2)$ and the actual rotation can be 8 degrees (an error of $-20 \%$ ).

A vehicle with $N$ steerable drive wheels has $N$ degrees of control for translation and $N$ degrees of control for steering. When the vehicle is moving in a circle, it has one degree of freedom. The instantaneous center of rotation determines the steering angles for the $N$ wheels. To control the vehicle motion, we introduce $N$ new variables: one variable for motion on the circle (the pseudovelocity) and $N-1$ variables for the errors in satisfying the rigid body constraints. To control the $N$ new variables, we introduce $N$ new control variables: on for the pseudovelocity 


\section{INTRODUCTION}

and $N-1$ forces to reduce the errors. We must define a mapping from the $N$ new control variables to the $N$ original control variables for translation.

Kankaanranta and Koivo ${ }^{4}$ have developed a general model for the dynamics of constrained mechanical systems and have proposed a control architecture that allows the force and position degrees of freedom to be decoupled. Their focus was on the constrained motion of manipulators and several recent papers ${ }^{1,7}$ have applied their results (or related results) to the constrained manipulator problem. We will apply their architecture to the constrained motion of a vehicle.

The next section will present a kinematic model of a vehicle with four steerable drive wheels. The third section will define the pseudovelocity for a vehicle with $N$ drive wheels and one degree of freedom. The fourth section will apply the control architecture of Kankaanranta and Koivo to a vehicle with $N$ drive wheels and one degree of freedom. The fifth section will discuss how to implement the control architecture (we have defined the force in terms of the error) and the sixth section will present experimental results using the HERMIES-III robot. The final section presents our conclusions. 


\section{KINEMATIC MODEL OF VEHICLE}

In this section, we will discuss the equations that comprise the kinematic model of the vehicle. The vehicle has four drive wheels that can be steered. The motion of each wheel is described by two variables: $w_{i}$, and $\theta_{i}$. The variable $w_{i}$ is the cumulative displacement of the wheel as it rotates about its axis. The variable $\theta_{i}$ is the steering angle of the wheel in the vehicle coordinates. The units for $w_{i}$ are meters, while the units for $\theta_{i}$ are radians.

Let $r_{i}$ be the radius from the instantaneous center of rotation $(P)$ to a wheel and let $v_{i}\left(\dot{w}_{i}\right)$ be the translational velocity of the wheel. If $\Omega$ is the rotation rate for the vehicle, then each wheel satisfies:

$$
v_{i} / r_{i}=\Omega
$$

The radii $\left(r_{i}\right)$ are determined by the center of rotation $\left[P=\left(P_{x}, P_{y}\right)\right]$ and by the location of each wheel $\left[{ }^{i} p=\left({ }^{i} p_{x},{ }^{i} p_{y}\right)\right]$. Let $\left(x_{i}, y_{i}\right)$ be the $x$ and $y$ components of the vector from wheel $i$ to the center of rotation in the vehicle coordinates:

$$
\begin{aligned}
& x_{i}=P_{x}-{ }^{i} p_{x} \\
& y_{i}=P_{y}-{ }^{i} p_{y}
\end{aligned}
$$

Then, the radii and wheel steering angles satisfy:

$$
\begin{gathered}
\left(x_{i}\right)^{2}+\left(y_{i}\right)^{2}=\left(r_{i}\right)^{2} \\
x_{i}=-r_{i} \sin \theta_{i} \\
y_{i}=r_{i} \cos \theta_{i}
\end{gathered}
$$

We assume that the wheels are numbered as in Fig. 1. Furthermore, we assume that the vectors from wheel 1 to wheel 3 and from wheel 2 to wheel 4 are parallel to the $x$ axis. Similarly, the vectors from wheel 1 to wheel 2 and from wheel 3 to wheel 4 are parallel to the $y$ axis. We assume that the origin of the vehicle coordinate system is at the midpoint of the four wheels. Thus, the coordinates of each wheel are:

$$
{ }^{1} p=(c,-d),{ }^{2} p=(c, d),{ }^{3} p=(-c,-d), \text { and }{ }^{4} p=(-c, d) .
$$

In Fig. 1, a vehicle with four wheels rotates about a point. During rotation the motion of the wheels must be synchronized. Using Eq. (1), the velocity of each wheel must be proportional to the radius from the wheel to the center of rotation. The four wheel velocities have one degree of freedom and must satisfy three rigid body constraints. We will now derive the rigid body constraints. 


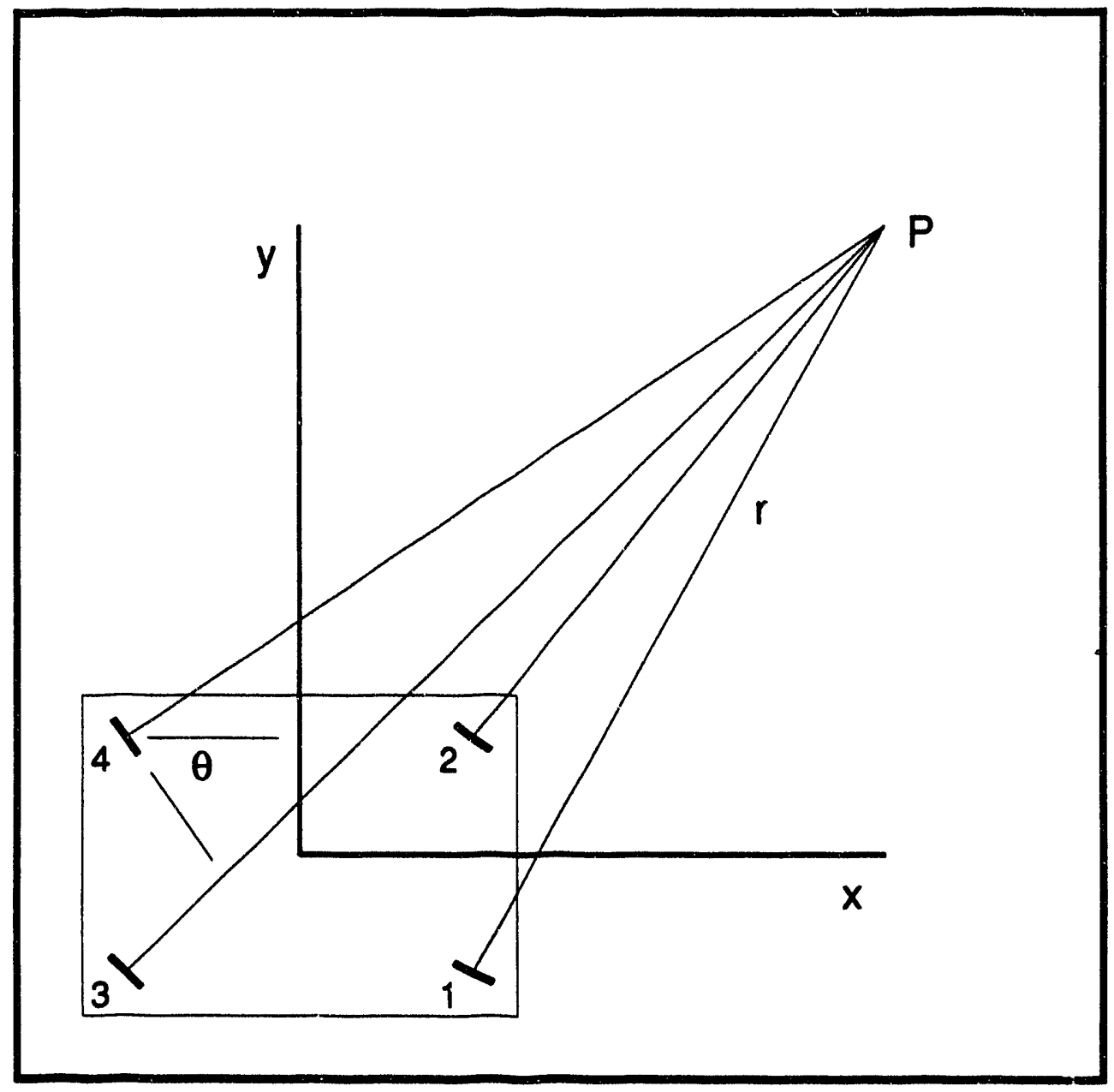

Fig. 1. A vehicle rotating about the Point $P$.

Given the center of rotation, we can calculate the four radii and the four steering angles using Eqs. (4) to (6). Alternatively, given steering angles for a pair of wheels and the distance between the wheels, we can calculate the distances from each wheel to $P$. We will consider four pairs of wheels (see Fig. 1): $[2,4],[1,3],[1,2]$, and $[3,4]$ and calculate four pairs of radii. For the wheels parallel to the $x$ axis $([2,4]$ and $[1,3])$ :

$$
\begin{aligned}
& r_{2}=2 c \cos \theta_{4} / \sin \left(\theta_{2}-\theta_{4}\right) \\
& r_{4}=2 c \cos \theta_{2} / \sin \left(\theta_{2}-\theta_{4}\right) \\
& r_{1}=2 c \cos \theta_{3} / \sin \left(\theta_{1}-\theta_{3}\right)
\end{aligned}
$$




$$
r_{3}=2 c \cos \theta_{1} / \sin \left(\theta_{1}-\theta_{3}\right)
$$

For the wheels parallel to the $y$ axis $([3,4]$ and $[1,2])$ :

$$
\begin{aligned}
& r_{3}=2 d \sin \theta_{4} / \sin \left(\theta_{4}-\theta_{3}\right) \\
& r_{4}=2 d \sin \theta_{3} / \sin \left(\theta_{4}-\theta_{3}\right) \\
& r_{1}=2 d \sin \theta_{2} / \sin \left(\theta_{2}-\theta_{1}\right) \\
& r_{2}=2 d \sin \theta_{1} / \sin \left(\theta_{2}-\theta_{1}\right)
\end{aligned}
$$

Using Eq. (1) and Eqs. (7) to (14), we can derive the following rigid body constraints:

$$
\begin{aligned}
& v_{1} \sin \theta_{1}=v_{2} \sin \theta_{2} \\
& v_{2} \cos \theta_{2}=v_{4} \cos \theta_{4} \\
& v_{4} \sin \theta_{4}=v_{3} \sin \theta_{3} \\
& v_{3} \cos \theta_{3}=v_{1} \cos \theta_{1}
\end{aligned}
$$

The physical significance of the rigid body constraints is that the velocity components along the line connecting the centers of each pair of wheels must be equal. Thus, the distance between wheels cannot change.

When a vehicle with four wheels is rotating about a point, the wheel velocities have one degree of freedom and must satisfy three rigid body constraints. Although Eqs. (15) to (18) provide four rigid body constraints, at most three of them can be independent. Which three of the four should we use? The answer depends on the steering angles. When the four wheels are pointed forward $\left(\theta_{i}=0\right)$, Eqs. (15) and (17) do not constrain the velocities. When the four wheels are pointed a ninety degrees, Eqs. (16) and (18) do not constrain the velocities. If we steer the front wheels (1 and 2) and the rear wheels are fixed in the forward position (the normal configuration for a car), Eq. (17) does not constrain the velocities. If we steer the rear wheels and the front wheels are fixed in the forward position (an appropriate choice when the vehicle moves backward), Eq. (15) does not constrain the velocities.

In the special cases when the four wheels are pointed forward or at ninety degrees, the wheel velocities have two degrees of freedom. The wheels are paired into two groups that must have the same speed. For forward wheels, wheels 1 and 3 have the same speed and wheels 2 and 4 have the same speed. Furthermore, wheels 1 and 2 can have different speeds. For wheels at 90 degrees, wheels 1 and 2 have the same speed and wheels 3 and 4 have the same speed. For these special cases, we 


\section{KINEMATIC MODEL OF VEHICLE}

have two sets of two wheels that each must satisfy a rigid body constraint. Thus, we can have two wheels with one constraint or four wheels with three constraints.

In all cases, we have $N$ wheels and one degree of freedom. 


\section{DEFINITION OF THE PSEUDOVELOCITY}

In the last section, we developed a kinematic model for a vehicle with four wheels. In this section, we will consider a vehicle with $N$ wheels and assume that it has one degree of freedom and $K$ degrees of constraint $(K+1=N)$. The general nonholonomic constraint may be written:

$$
A \dot{q}=0
$$

where the $q$ are the generalized coordinates. We assume that the $K \times N$ matrix $A$ has full rank $K$. For the vehicle, we will let the $q$ be the rotation of the four wheels $(q=w)$ and we assume that the steering angles are exogenous functions of time. Naturally, the steering angles are not independent; the center of rotation $(P)$ determines all of the steering angles. Since the matrix $A$ is function of the steering angles, it is a known function of time.

Following Kankaanranta and Koivo, we introduce a scalar pseudovelocity $(v)$ :

$$
v=B \dot{q}
$$

where $B$ is a $1 \times N$ matrix, chosen to make $\left[A^{T} \quad B^{T}\right]^{T}$ nonsingular. The vehicle has $N$ wheels and one degree of freedom. The pseudovelocity has one degree of freedom and determines the motion of the vehicle. The velocities of all $N$ wheels $(\dot{q})$ will be proportional to the pseudovelocity.

Kankaanranta and Koivo do not specify a method for choosing $B$. Many choices are possible. Some authors have chosen the pseudovelocity to be a physical velocity (for example, the velocity of one of the wheels). One of our major contributions to this problem is that we have developed a general method for choosing $B$. We choose $B$ to be orthogonal to all of the rows of $A$.

Let $S$ be the composite matrix with its first $K$ rows from $A$ and its last row from $B$ :

$$
S=\left[\begin{array}{cccc}
a_{11} & a_{12} & \ldots & a_{1 N} \\
a_{21} & a_{22} & \ldots & a_{2 N} \\
\cdot & \cdot & \cdot & \cdot \\
\cdot & \cdot & \cdot & \cdot \\
a_{K 1} & a_{K 2} & \ldots & a_{K N} \\
b_{1} & b_{2} & \ldots & b_{N}
\end{array}\right]
$$

Expanding along the last row of $S$, the determinant of $S$ is:

$$
|S|=\sum_{i=1}^{N} b_{i} \Delta_{N i}
$$

where the $\Delta_{N i}$ are the cofactors of $S$. Since the cofactors depend on the elements of $A$, they are functions of the steering angles $\left(\theta_{i}\right)$. 
The inverse of $S$ is partitioned into two matrices $(E$ and $F)$ :

$$
S^{-1}=\left[\begin{array}{ll}
E & F
\end{array}\right]
$$

where $E$ is an $(N \times K)$ matrix and $F$ is an $(N)$ vector. The matrices $A, B, E$, and $F$ satisfy:

$$
A E=I, A F=0, B E=0, B F=1 \text {, and } E A+F B=I \text {. }
$$

The vector $F$ is the orthogonal complement of $A$ and is given by:

$$
F=\frac{1}{|S|}\left[\begin{array}{c}
\Delta_{N 1} \\
\Delta_{N 2} \\
\cdot \\
\cdot \\
\Delta_{N N}
\end{array}\right]
$$

Equation (24) is a general expression for $F$ that is valid for any choice for the $B$ vector. The cofactors are functions of the elements of $A$. The elements of the vector $B$ are in the determinant of $S$.

How shall we define the $B$ vector? Our goal is to make the matrix $S$ nonsingular (to make the determinant of $S$ nonzero). If we choose the $b_{i}$ to be proportional to $\Delta_{N i}$, the determinant of $S$ cannot be zero unless all of the cofactors are equal to zero. Furthermore, the $B$ vector will be parallel to the $F$ vector. Thus, the $B$ vector will be orthogonal to all of the rows of $A$. We choose the $b_{i}$ to be proportional to $\Delta_{N i}$ and we introduce a normalization factor to make $|S|=1$. Define the normalization factor $(\rho)$ by:

$$
\rho=\sum_{i=1}^{N}\left(\Delta_{N i}\right)^{2}
$$

Thus, our choices for the elements of the $B$ vector are:

$$
b_{i}=\Delta_{N i} / \rho
$$

The vector $B$ and the wheel velocities $(\dot{q})$ define the pseudovelocity. In general, the pseudovelocity will not be the velocity of any particular point on the vehicle. However, the pseudovelocity determines the motion of the vehicle (the wheel velocities are proportional to the pseudovelocity). The pseudovelocity can be controlled to follow a reference trajectory. Given planned trajectories for the wheel velocities and steering angles, the reference trajectory for the pseudovelocity is determined by Eq. (20).

To illustrate our method for choosing the $B$ vector, we will consider the example of a vehicle that has both front and rear wheel steering. We assume that most of the steering is with the front wheels and that the rear-wheels are normally in the forward position, where Eq. (17) does not constrain the velocities. If we exclude 
Eq. (17) and define the rows of the $A$ matrix by Eqs. (15), (16), and (18), the $S$ matrix is:

$$
S=\left[\begin{array}{cccc}
s_{1} & -s_{2} & 0 & 0 \\
0 & c_{2} & 0 & -c_{4} \\
-c_{1} & 0 & c_{3} & 0 \\
b_{1} & b_{2} & b_{3} & b_{4}
\end{array}\right]
$$

where $s_{i}=\sin \theta_{i}$ and $c_{i}=\cos \theta_{i}$. Whenever the rear wheels are in the forward position, the $S$ matrix will not be singular unless the front wheels are also in the forward position.

For this example, the cofactors of $S$ are:

$$
\begin{aligned}
& \Delta_{41}=s_{2} c_{3} c_{4} \\
& \Delta_{42}=s_{1} c_{3} c_{4} \\
& \Delta_{43}=c_{1} s_{2} c_{4} \\
& \Delta_{44}=s_{1} c_{2} c_{3}
\end{aligned}
$$

Given the cofactors, the elements of the $B$ vector are determined by Eqs. (25) and (26).

For the case of two constrained wheels, we will introduce a norms lization factor $(\mu)$ for the elements of the $A$ matrix. The rigid body constraint for a vehicle with two wheels is Eq. (15). Using the normalization factor:

$$
A=\left[\beta_{1}-\beta_{2}\right]
$$

where $\beta_{i}=s_{i} / \mu$ and

$$
\mu^{2}=\left(s_{1}\right)^{2}+\left(s_{2}\right)^{2}
$$

The rank of $A$ is one unless both of the steering angles are zero (the vehicle is not constrained). For this example, the cofactors are: $\Delta_{21}=\beta_{2}$ and $\Delta_{22}=\beta_{1}$. Since $\left(\beta_{1}\right)^{2}+\left(\beta_{2}\right)^{2}=1$, the normalizatior constant $(\rho)$ is unity and $b_{i}=\Delta_{2 i}$. Thus, the $S$ matrix is:

$$
S=\left[\begin{array}{cc}
\beta_{1} & -\beta_{2} \\
\beta_{2} & \beta_{1}
\end{array}\right]
$$

For this case, the $S$ matrix is orthogonal. 


\section{POSITION AND FORCE CONTROL ARCHITECTURE}

A vehicle with $\mathrm{N}$ steerable drive wheels has $\mathrm{N}$ degrees of control for translation and $N$ degrees of control for steering. When the vehicle is moving in a circle, it has one degree of freedom. The instantaneous center of rotation determines the steering angles for the $N$ wheels. In our previous work, ${ }^{6}$ we planned reference trajectories for the $2 N$ state variables that satisfied the rigid body constraint [Eq. (19)] and had independent control for each wheel. However, we found significant errors during constrained motion. In this section, we make the novel proposal that force control must be added to position control when a vehicle is moving in a circle.

We will use a kinematic model of the vehicle rather than a dynamic model. Since most of the theory of constrained mechanical systems is based on dynamics, we will briefly justify our choice. We have two basic lines of argument: a kinematic model is less complex than a dynamic model and our goal is to control the kinematic variables (position, velocity, and acceleration). A realistic dynamic model of a vehicle would be very complex and would require a detailed map of the mass distribution. Furthermore, the mass distribution can change with time (HERMIES-III carries a manipulator that weighs $160 \mathrm{~kg}$ ). We start with simple models and move to complex models when the simple models fail. For our vehicle, a kinematic model is sufficient. A system that has significant changes in mass distribution or moment of inertia might require a dynamic :odel.

The sensors on our vehicle measure position (wheel translation and steering angle). We do not have any sensors that measure forces or torques on the wheels. Thus, the appropriate variables for feedback control are the measured variables. And the appropriate model is a kinematic model.

Following Goldstein,$^{2}$ the equations of motion for the constrained system are:

$$
\ddot{q}=\tau+A^{T} \lambda
$$

where $\tau$ is a vector of acceleration inputs and $\lambda$ is a vector Lagrange multiplier with $K$ components. In the unconstrained case $(A=0)$, each wheel has an independent acceleration that is controlled by $\tau$. In the constrained case, the accelerations are coupled. If the acceleration is zero for all wheels but one, all of the wheels will feel the acceleration through the last term $\left(A^{T} \lambda\right)$. Goldstein and Kankaanranta and Koivo both say that the term $\left(A^{T} \lambda\right)$ may be identified with the generalized force of constraint acting on the system. We will refer to the elements of $\lambda$ as forces (although they are really accelerations). In our previous work, we neglected the forces and controlled the wheels independently.

Building on the seminal work of Hemami and Weimer, ${ }^{3}$ Kankaanranta and Koivo define the control architecture by:

$$
\tau=A^{T} u_{1}+F u_{2}-(E \dot{A}+F \dot{B}) F v
$$


where $u_{1}$ is a vector with $K$ components and $u_{2}$ is a scalar. For this architecture, the force and position degrees of freedom are decoupled:

$$
-\lambda=u_{1} \quad \dot{v}=u_{2} .
$$

$u_{1}$ controls the force while $u_{2}$ controls the position.

The vehicle has $N$ acceleration inputs $(\tau)$ that control translation. We have introduced $N$ new control variables $\left(u_{1}\right.$ and $u_{2}$ ). Equation (36) provides a mapping from the new control variables to the original control variables. One of the new variables controls the pseudovelocity, while the remaining variables control the forces. In the next section, we will relate the forces to errors.

While Eq. (36) is correct, the third term on the right can be simplified. Combining Eq.s. (19) and (20):

$$
S \dot{q}=\left[\begin{array}{l}
0 \\
v
\end{array}\right]
$$

Using Eq. (23) to solve for $\dot{q}$ :

$$
\dot{q}=F v
$$

If we differentiate Eq. (39):

$$
\ddot{q}=F \dot{v}+\dot{F} v
$$

Kankaanranta and Koivo's expression for $\ddot{q}$ is:

$$
\ddot{q}=F \dot{v}-(E \dot{A}+F \dot{B}) F v
$$

Comparing Eqs. (40) and (41), we find:

$$
\dot{F} v=-(E \dot{A}+F \dot{B}) F v
$$

Using Eq. (42), Eq. (36) may be simplified:

$$
\tau=A^{T} u_{1}+F u_{2}+\dot{F} v
$$

For some choices of the $B$ matrix, calculation of the right side of Eq. (42) might be easier than the calculation of the left side. However for our choice for the $B$ matrix, the derivative of $F$ is less complex than the derivative of $B$ and Eq. (43) requires fewer calculations than Eq. (36). To demonstrate the advantages of Eq. (43), we will derive expressions for $\dot{B}$ and $E$. Equation (26) may be written:

$$
B=F^{T} / \rho
$$

The derivative of Eq. (44) is:

$$
\dot{B}=\dot{F}^{T} / \rho-F^{T}\left(\dot{\rho} / \rho^{2}\right)
$$


Since $E A+F B=I$,

$$
E A A^{T}+F B A^{T}=A^{T}
$$

Since $B A^{T}=0$, the second term on the left side is zero. Since $A A^{T}$ is positive definite, it is nonsingular and Eq. (46) can be solved for $E$ :

$$
E=A^{T}\left(A A^{T}\right)^{-1}
$$




\section{POSITION AND FORCE CONTROL - IMPLEMENTATION}

Kankaanranta and Koivo define a control architecture that decouples the force and position degrees of freedom. However, they did not specify the force. In this section, we will define an error vector $(e)$ and define the force in terms of the error.

The feedbacks from the vehicle are the encoder readings for wheel rotation and steering angle $\left(w_{i}\right.$ and $\left.\theta_{i}\right)$. Given two measurements of wheel rotation for a wheel and the time between the measurements, we can calculate a measured average velocity $(\dot{q})$ for each wheel. Using Eq. (20), we can calculate the pseudovelocity $(v)$. Either the measured values or the target values of the steering angles can be used to calculate the matrices $A, B$, and $F$ (we do not need to calculate the $E$ matrix). In the experimental results (reported in the next section), we have used the target values.

The kinematic model of the vehicle has a system of general nonholonomic constraints [Eq. (19)]. Hov ever, the measured average velocities may not satisfy the constraints. We will deine an error vector with $K$ components by:

$$
\dot{e}=A \dot{q}
$$

where the initial condition is $e_{i}(0)=0$. We will use the force vector $\left(u_{1}\right)$ to drive the error to zero:

$$
u_{1 i}=k_{1 i} \dot{e}_{i}+k_{2 i} e_{i} \text { for } i=1 \text { to } K
$$

In Eq. (49), we have defined the force vector using measurements from the wheel encoders. We have not needed to use force sensors to provide force feedback. Thus, we have not needed to add expensive force sensors to implement force control of the vehicle.

To control the pseudovelocity $(v)$, we assume that $v$ is tracking a target $\left(v^{G}\right)$. We will define the velocity error $(\epsilon)$ by:

$$
\dot{\epsilon}=v^{G}-v
$$

where the initial condition is $\epsilon(0)=0$. We will use the pseudovelocity $\left(u_{2}\right)$ control to drive the velocity error to zero:

$$
u_{2}=k_{3} \dot{\epsilon}+k_{4} \epsilon
$$

The target for the pseudovelocity is determined by the targets for the wheels:

$$
v^{G}=B \dot{q}^{G}
$$

At the end of an experiment, the vehicle stops moving but the forces will not be zero unless the cumulative errors $(e)$ are zero. To avoid jerk when power is removed from the vehicle, the forces should gracefully decay to zero at the end of an experiment. To implement this policy, the cumulative errors exponentially decay 


\section{POSITION AND FORCE CONTROL - IMPLEMENTATION}

whenever the pseudovelocity target is zero. Thus, when $v^{G}=0$, an extra term is added to Eq. (48):

$$
\dot{e}=A \dot{q}-\alpha e
$$

where $\alpha$ is a positive constant (for our experimental results, $\alpha=10$ ). 


\section{EXPERIMENTAL RESULTS}

To perform experiments, we modified the existing wheel control system for the HERMIES-III robot. ${ }^{6}$ The modifications were to two modules: Wheel Driver and Motor Driver. The Wheel Driver reads a Wheel Target and a Wheel Control from shared memory and sends a Setpoint and a Rate to the Motor Driver. The Motor Driver sends the torque signals to the motors and returns encoder readings to the Wheel Driver. The Wheel Target values are the translational velocities and steering angles of the two wheels $\left(v_{i}\right.$ and $\left.\theta_{i}\right)$. The Wheel Control values are the rotational accelerations and steering velocities.

The Wheel Driver performs calculations at about $20 \mathrm{Hertz}$. The Wheel Driver calculations can use floating point numbers and physical units (meters, radians, and seconds). The Motor Driver is guaranteed to perform calculations at $100 \mathrm{Hertz}$; it cannot use floating point numbers but must use integers (the floating point registers are not saved by the interrupt service routine). The units for the Motor Driver are encoder clicks and clock ticks ( 0.01 second).

The modified inputs to the Motor Driver are three two dimensional arrays of data: $s p, s p . . d o t$, and sine. The setpoint $(s p)$ is the next target for the variable. The units for $s p$ are encoder clicks per tick for wheel rotation and clicks for wheel steering. The array $s p_{\text {_d dot }}$ is the rate of change in the setpoint allowed in a tick. The array sine is the only new variable that we have introduced to control two constrained wheels. When the wheels are unconstrained, sine $[i]=0$. When the wheels are constrained, sine $[i]=8192 * \beta_{i}$ [where $\beta_{i}$ is defined after Eq. (32)].

What is the reason for the factor 8192 ? Since $\beta_{i}$ is a floating point number that is less than (or equal to) 1.0 and sine is an integer, a scale factor is required. Integers have 32 bits. To prevent overflow during multiplication of two integers, each integer should have less than 16 bits. If we reserve one bit for sign, the maximum scale factor would be $\left(2^{15}-1\right)=32,767$. How accurately can we measure the steering angles? Do we need such a large scale factor? The maximum steering angle (90 degrees) corresponds to 16,384 encoder clicks. However, the increment in encoder readings is 16 . Thus, the accuracy is limited to 1024 distinct values. The value 8192 is eight times the accuracy. The value 8192 requires 14 bits. Thus, the scale figure provides sufficient accuracy and is a factor of four below the maximum scale factor.

To demonstrate the benefits of the new control system for constrained wheels, we performed two sets of experiments. The first experiment was a rotation of 120 degrees about the point $(2,2)$ in the vehicle coordinate system. Figure 1 shows the vehicle coordinate system for a vehicle with four wheels. The experiments use a vehicle with two wheels and the origin of the vehicle coordinate system is midway between the two wheels. The second experiment was a straight movement of ten feet with the wheels at a 30 degree angle in the vehicle coordinate system. For both experiments, the steering angles were constant during the motion.

To perform an experiment, we use a program that reads a data set and writes a sequence of Wheel Targets and Wheel Controls to shared memory where they 


\section{EXPERIMENTAL RESULTS}

are read by the Wheel Driver and used to calculate the input arrays for the Motor Driver.

The input data set for a positive (counterclockwise) rotation of 120 degrees about the point ( 2 meters, 2 meters) is displayed in Table 1. The first three rows of Table 1 contain five values: time (measured in seconds), targets for the wheel velocities $\left(v_{1}\right.$ and $\left.v_{2}\right)$, and targets for the steering angles $\left(\theta_{1}\right.$ and $\left.\theta_{2}\right)$. The units of the wheel velocities are meters/second and the units of the steering angles are radians.

Table 1. Input data for a positive rotation of $\mathbf{1 2 0}$ degrees about (2 meters, 2 meters)

\begin{tabular}{|c|c|c|c|c|}
\hline Time & $v_{1}$ & $v_{2}$ & $\theta_{\mathrm{i}}$ & $\theta_{2}$ \\
\hline 2.50 & 0.0 & 0.0 & -0.6985 & -0.8905 \\
\hline 14.47 & 0.45 & 0.3723 & -0.6985 & -0.8905 \\
\hline 5.15 & 0.0 & 0.0 & -0.6985 & -0.8905 \\
\hline
\end{tabular}

The distance between the two wheels is 0.7632 meters (to avoid roundoff error, we will display results to four figures). The steering angles are given by:

$$
\begin{aligned}
\tan \theta_{1} & =-2 /(2+0.3816) \\
\tan \theta_{2} & =-2 /(2-0.3816) .
\end{aligned}
$$

The radii to the center of rotation $\left(r_{1}\right.$ and $\left.r_{2}\right)$ are determined by:

$$
\begin{aligned}
& \left(r_{1}\right)^{2}=2^{2}+(2+0.3816)^{2} \\
& \left(r_{2}\right)^{2}=2^{2}+(2-0.3816)^{2}
\end{aligned}
$$

Thus,

$$
\begin{aligned}
& r_{1}=3.1100 \\
& r_{2}=2.5728
\end{aligned}
$$

The ratios of the wheel velocities are given by Eq. (15):

$$
\left(v_{1} / v_{2}\right)=\sin \theta_{2} / \sin \theta_{1}=1.2088
$$


The velocity target for the right wheel is 0.45 meters per second. The velocity target for the left wheel ( 0.3723 meters per second) is determined by dividing 0.45 by the velocity ratio. The acceleration rate for the left wheel $\left(0.1735\right.$ meters per second $\left.{ }^{2}\right)$ is determined by dividing the acceleration rate for the right wheel $(0.2097$ meters per second ${ }^{2}$ ) by the velocity ratio.

During the first segment of the experiment, the wheels are steered while the vehicle is at rest. At the steering velocities for the vehicle (1.0546 radians per second), the steering motion should take less than one second. However, the length of the first segment is 2.5 seconds. The extra time allows the steering motion to be completed before the wheels begin to rotate.

During the second segment of the experiment, the wheels accelerate to maximum velocity. During the rotation motion, the right wheel will travel 6.51 meters. The time required to reach maximum speed is 2.15 seconds. During that time, the right wheel will travel 0.48 meters. The right wheel will ramp up for 0.48 meters, travel at full speed for 5.55 meters, and ramp down for 0.48 meters. The right wheel requires 12.32 seconds to travel 5.55 meters. Thus, the velocity target for the right wheel should be 0.45 meters/second for 14.47 seconds (the sum of 2.15 and 12.32) and should be 0.0 for 2.15 seconds. We have added 3 seconds to the final segment to allow the force to decay.

The input data set for a forward movement of 10 feet at a 30 degree angle is displayed in Table 2. The steering angles for the two wheels are the same (30 degrees $=0.5236$ radians). The rampup time and distance are the same as in the first experiment. Each wheel will travel 3.05 meters (10 feet). Each wheel will ramp up for 0.48 meters, travel at full speed for 2.08 meters, and ramp down for 0.48 meters. Each wheel requires 4.62 seconds to travel 2.08 meters. Thus, the velocity target for the wheels should be 0.45 meters/second for 6.77 seconds (the sum of 2.15 and 4.62). The initial and final segments are similar to the first experiment.

Table 2. Input data for a forward movement of 10 feet at 30 degrees

\begin{tabular}{|c|c|c|c|c|}
\hline Time & $v_{2}$ & $v_{1}$ & $\theta_{2}$ & $\theta_{1}$ \\
\hline 3.00 & 0.0 & 0.0 & 0.5236 & 0.5236 \\
\hline 6.77 & 0.45 & 0.45 & 0.5236 & 0.5236 \\
\hline 5.14 & 0.0 & 0.0 & 0.5236 & 0.5236 \\
\hline
\end{tabular}


When the experiments are performed, detailed records of the values of the key variables are stored in data sets. Both experiments were performed four times. The results of the two experiments are summarized in Table 3.

Table 3. Summary of the results for two experiments

\begin{tabular}{|c|c|c|c|c|c|}
\hline Exp. & Case & Direction & Force & Ratio & Error (\%) \\
\hline 1 & 1 & Positive & No & 1.1424 & -5.49 \\
\hline 1 & 2 & Negative & No & 1.1409 & -5.61 \\
\hline 1 & 3 & Positive & Yes & 1.2064 & -0.20 \\
\hline 1 & 4 & Negative & Yes & 1.2068 & -0.17 \\
\hline 2 & 5 & Backward & No & 1.0822 & 8.22 \\
\hline 2 & 6 & Forward & No & 1.0898 & 8.98 \\
\hline 2 & 7 & Backward & Yes & 1.0009 & 0.09 \\
\hline 2 & 8 & Forward & Yes & 1.0015 & 0.15 \\
\hline
\end{tabular}

The four cases for the first experiment are numbered: Case 1 to Case 4. The direction of the rotation alternates between positive and negative. For Cases 1 and 2 , the force control was turned off by setting the gain parameters to zero. For Cases 3 and 4, the force cortrol was active. The ratio of the total movement of the right wheel to the total movement of the left wheel $\left(w_{1} / w_{2}\right)$ should be equal to the ratio of the velocities $\left(v_{1} / v_{2}=1.2088\right)$. Without force control, the errors are about $5.5 \%$. With force control, the error is reduced by more than a factor of 20 to about $0.2 \%$.

The four cases for the second experiment are numbered: Case 5 to Case 8. The force control was off for Cases 5 and 6 and on for Cases 7 and 8. For this experiment, the rotation ratio should be 1.0. Without force control, the errors are more than $8 \%$. With force control, the error is reduced by more than a factor of 40 to less than $0.2 \%$.

The rotation ratios for the two experiments are displayed in Figs. 2 and 3. After the initial transient, the rotation ratio rapidly approaches a constant value for all eight cases. 


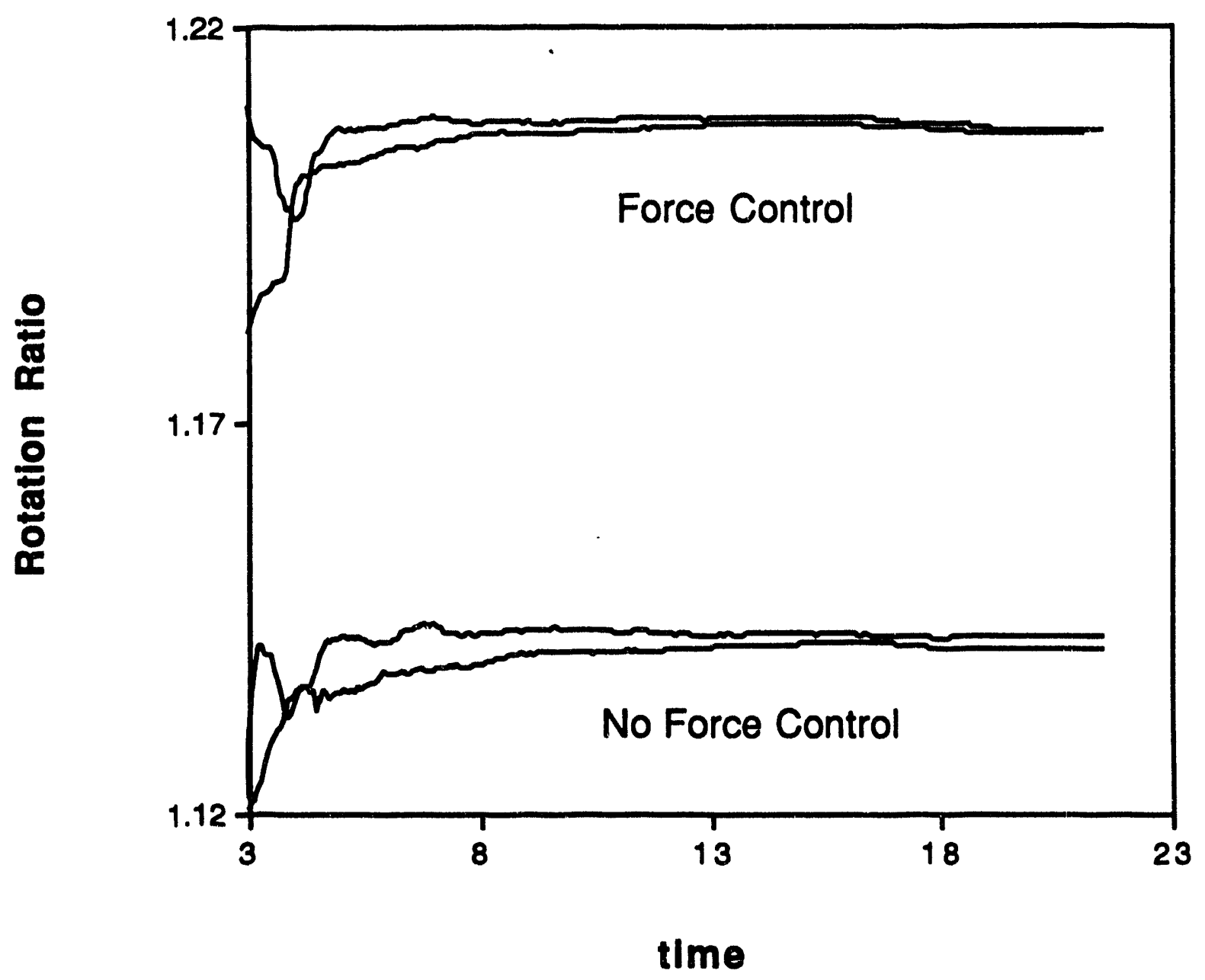

Fig. 2. The rotation ratio for the first experiment (the goal is 1.2088). 


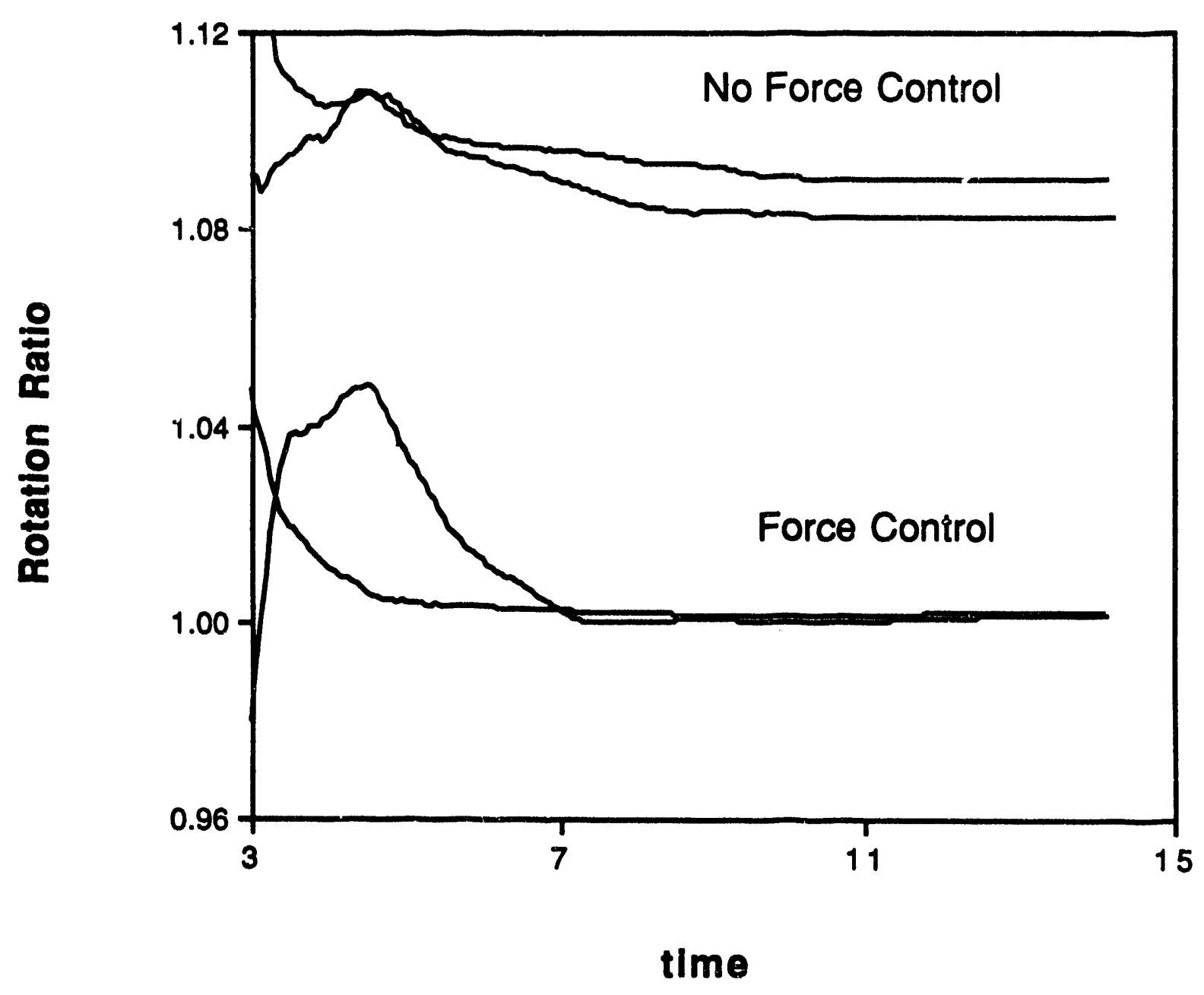

Fig. 3. The rotation ratio for the second experiment (the goal is 1.0). 
The control signals $\left(u_{1}\right.$ and $\left.u_{2}\right)$ are displayed in Fig. 4 for Case 3. The control signals in the figure are dimensionless integers that have been divided by 10,000 . The control signal for position and pseudovelocity $\left(u_{2}\right)$ is large and positive during the initial startup and large and negative during the final rampdown. During the constant velocity phase of the motion, $u_{2}$ is much smaller. The control signal for force and error reduction $\left(u_{1}\right)$ becomes large during the startup phase, remains at a high level during the middle phase, and becomes very large during the rampdown phase. At the end of the experiment, the control signals (and resultant forces) exponentially decay to zero [the decay is the result of Eq. (53)].

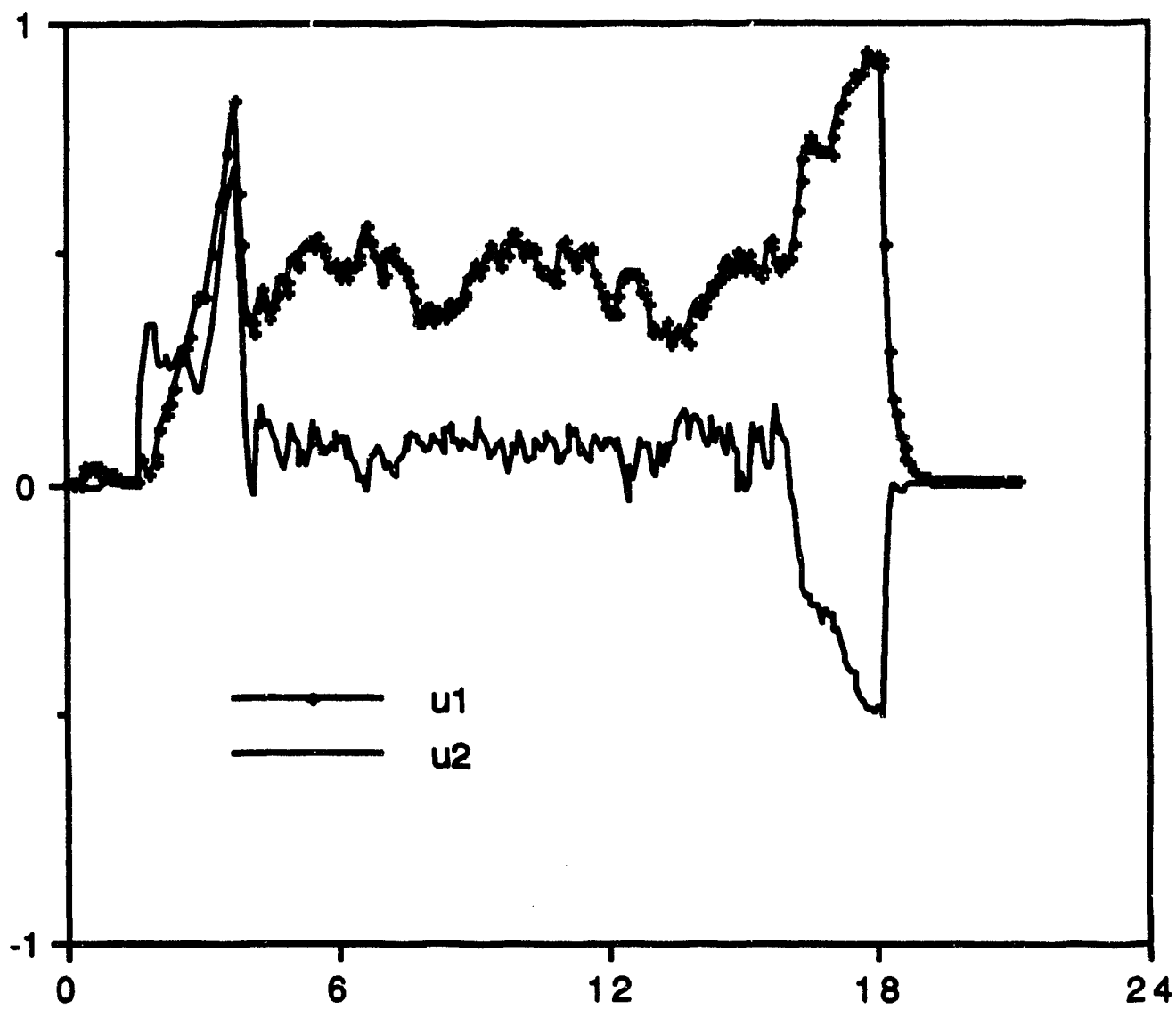

time

Fig. 4. The control signals ( $u_{1}$ and $u_{2}$ ) for Case 3. 
The output torque values are displayed in Fig. 5 for Case 3. In the notation of Fig. 5: $\tau_{1}=t R$ and $\tau_{2}=t L$. The torque values in the figure are dimensionless integers that have been divided by 10,000 . To prevent damage to the motors, the magnitude of the torque values is required to be less than 32,768 . For a short initial period, both torque values are positive. Soon, the right values are positive and the left values are negative. Thus, during most of the motion the two wheels are pulling against each other.

ํㅜㄴ

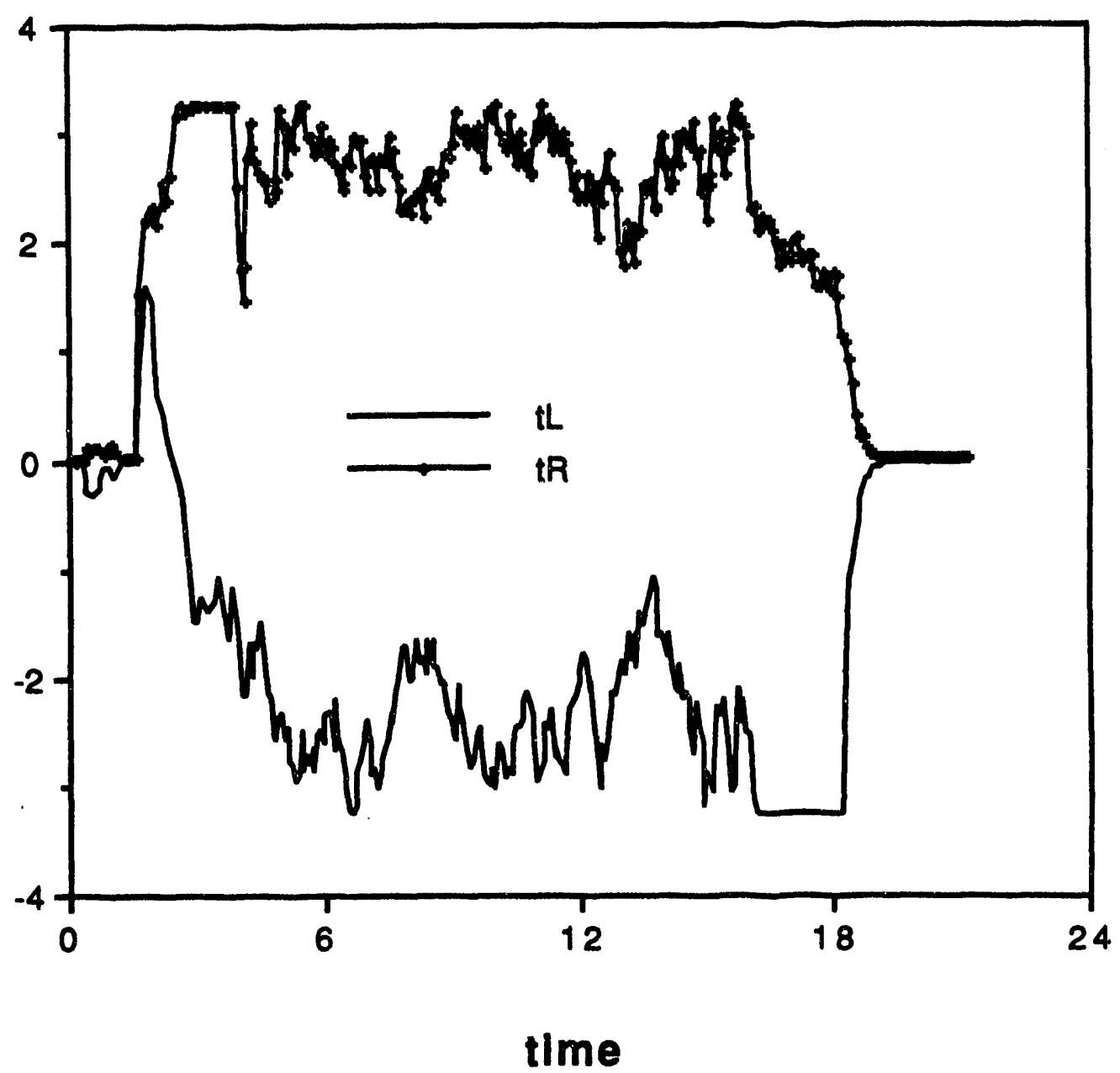

Fig. 5. The output torque values $\left(\eta_{1}=t R\right.$ and $\left.\eta_{2}=t L\right)$ for Case 3 . 


\section{CONCLUSIONS}

Wher a vehicle with two or more steerable drive wheels is traveling in a circle, the motion of the wheels is constrained. When the drive wheels are controlled independently, errors may occur and the wheels may slip. A vehicle with $N$ drive wheels has $(N-1)$ rigid br ly constraints and one degree of freedom. To control the vehicle, we have one degre of freedom for the position on the circle and $(N-1)$ forces that can be used to reduce errors. Kankaanranta and Koivo have developed a control architecture that allows the force and position degrees of freedom to be decoupled.

Kankaanranta and Koivo did not define a method for choosing the $B$ matrix that determines the pseudovelocity. We have developed a general method for choosing $B$ for a system with $N$ velocities and $N-1$ constraints. We choose $B$ to be orthogonal to all of the rows of the constraint matrix $(A)$. Furthermore, our choice produces a simple analytical expression for the orthogonal complement of $A$.

In addition to defining the $B$ matrix, we have made several modifications to the work of Kankaanranta and Koivo. We have used a kinematic model of the vehicle rather than a dynamic model. We have simplified the expression for the control architecture. We have used the constraint matrix to define an error vector and have defined the forces in terms of the errors. At the end of an experiment, the vehicle stops moving but the forces will not be zero unless the cumulative errors are zero. To have the forces gracefully decay to zero, the cumulative errors exponentially decay whenever the pseudovelocity target is zero.

We have implemented the control architecture on the HERMIES-III robot and have performed two sets of experiments. The implementation of force control is based on measurements from wheel encoders and does not require force sensors. The first experirnent was a rotation of 120 degrees about the point $(2$ meters, 2 meters). The second experiment was a movement of ten feet at a 30 degree angle. For both experiments, the reference values for the steering angles were constant during the motion. We have measured a dramatic reduction in error (more than a factor of $\mathbf{2 0}$ for the first experiment and more than a factor of 40 for the second experiment) compared to motions without force control. 


\section{REFERENCES}

1. H. Faessler, "Manipulators Constrained by Stiff Contact: Dynamics, Control, and Experiments," Int. J. Robotics Res. 9(4), 40-58 (1990).

2. H. Goldstein, Classical Mechanics, Reading, Massachusetts: Addison-Wesley (1980).

3. H. Hemami and F. C. Weimer, "Modeling of Nonholonomic Dynamic Systems with Applications," ASME J. Appl. Mech. 48, 177-182 (March 1981).

4. R. K. Kankaanranta and H. N. Koivo, "Dynamics and Simulation of Compliant Motion of a Manipulator," IEEE J. Robotics Automat. RA-4(2), 163-173 (April 1988).

5. N. Matsumoto and M. Tomizuka, "Vehicle Lateral Velocity and Yaw Rate Control with Two Independent Control Inputs," Proc. of the 1990 American Control Conference, 1868-75 (1990).

5. D. B. Reister, "A New Wheel Control System for the Omnidirectional HERMIES-III Robot," Robotica 10, 351-360 (1992).

7. M. A. Unseren, "Rigid Body Dynamics and Decoupled Control Architecture for Two Strongly Interacting Manipulators," Robotica 9, 421-430 (1991). 


\section{INTERNAL DISTRIBUTION}

1. B. R. Appleton

2. J. E. Baker

3. A. L. Bangs

4. M. Beckerman

5. B. L. Burks

6. R. J. Carter

7. J. R. Einstein

8. C. W. Glover

9. X. Guan

10. W. R. Hamel

11. J. N. Herndon

12. J. F. Jansen

13. J. P. Jones

14. H. E. Knee

15. R. L. Kress

16-20. R. C. Mann

21. E. M. Oblow
22-26. F. G. Pin

27-31. D. B. Reister

32. J. C. Schryver

33. P. F. Spelt

34. F. J. Sweeney

35. E. C. Uberbacher

36. M. A. Unseren

37-41. R. C. Ward

42. EPMD Reports Office

43-44. Laboratory Records Department

45. Laboratory Records, ORNL-RC

46. Document Reference Section

47. Central Research Library

48. ORNL Patent Section

\section{EXTERNAL DISTRIBUTION}

49. Dr. Peter Allen, Department of Computer Science, 450 Computer Science, Columbia University, New York, NY 10027

50. Mr. Harry Alter, Division of Advanced Technology Development, U.S. Department of Energy, Washington, DC 20545

51. Dr. John Baillieul, Aerospace and Mechanical Engineering Department, Boston University, 110 Cummington St., Boston, MA 02215

52. Dr. Wayne Book, Department of Mechanical Engineering, J. S. Coon Building, Room 306, Georgia Institute of Technology, Atlanta, GA 30332

53. Dr. Johann Borenstein, The University of Michigan, 1101 Beal Ave., Ann Arbor, MI 48109-2110

54. Professor Roger W. Brockett, Wang Professor of Electrical Engineering and Computer Science, Division of Applied Sciences, Harvard University, Cambridge, MA 02138

55. Professor Carl Crane, 202 Nuclear Science Center, University of Florida, Gainesville, FL 32611

56. Professor John J. Dorning, Department of Nuclear Engineering and Physics, Thornton Hall, McCormick Rd., University of Virginia, Charlottesville, VA 22901

57. Dr. Steven Dubowsky, Massachusetts Institute of Technology, Building 3, Room 469A, 77 Massachusetts Ave., Cambridge, MA 02139

58. Dr. Ralph C. Gonzalez, Department of Electrical and Computer Engineering, The University of Tennessee, Knoxville, TN 37996-2100

59. Dr. Avi Kak, Department of Electrical Engineering, Purdue University, Northwestern Ave., Engineering Mall, Lafayette, IN 47907

00. Professor Takeo Kanade, Computer Science and Robotics, Carnegie Mellon University, Pittsburgh, PA 15213-3890

61. Dr. James E. Leiss, Route 2, Box 142C, Broadway, VA 22815 
62. Professor Suzanne M. Lenhart, Department of Mathematics, The University of Tennessee, 304 Ayres Hall, Knoxville, TN 37996-1300

63. Dr. Oscar P. Manley, Division of Engineering, Mathematical, and Geosciences, Office of Basic Energy Sciences, ER-15, U.S. Department of Energy - Germantown, Washington, DC 20545

64. Professor Neville Moray, Department of Mechanical and Industrial Engineering, University of Illinois, 1206 West Green St., Urbana, IL 61801

65. Dr. Shankar Shastry, Department of EECS, 261-M Cory Hall, University of California, Berkeley, CA 94720

66. Dr. Wes Snyder, Department of Radiology, Bowman Gray School of Medicine, 300 S. Hawthorne Dr., Winston-Salem, NC 27103

67. Professor Delbert Tesar, Department of Mechanical Engineering, 26 San Jacinto, University of Texas, Austin, TX 78712

68. Professor M. A. Trivedi, Department of Electrical and Computer Engineering, The University of Tennessee, Knoxville, TN 37996-2100

69. Professor James S. Tulenko, 202 Nuclear Science Center, University of Florida, Gainesville, FL 32611

70. Professor David K. Wehe, Department of Nuclear Engineering, 2355 Bonisteel Blvd., The University of Michigan, Ann Arbor, MI 48109-2106

71. Professor Mary F. Wheeler, Department of Mathematical Sciences, Rice University, P.O. Box 1892, Houston, TX 77251

72. Office of Assistant Manager for Energy Research and Development, U.S. Department of Energy, Oak Ridge Operations Office, P.O. Box 2001, Oak Ridge, TN 37831-8600

73-82. Office of Scientific Technical Information, P.O. Box 62, Oak Ridge, TN 37831 

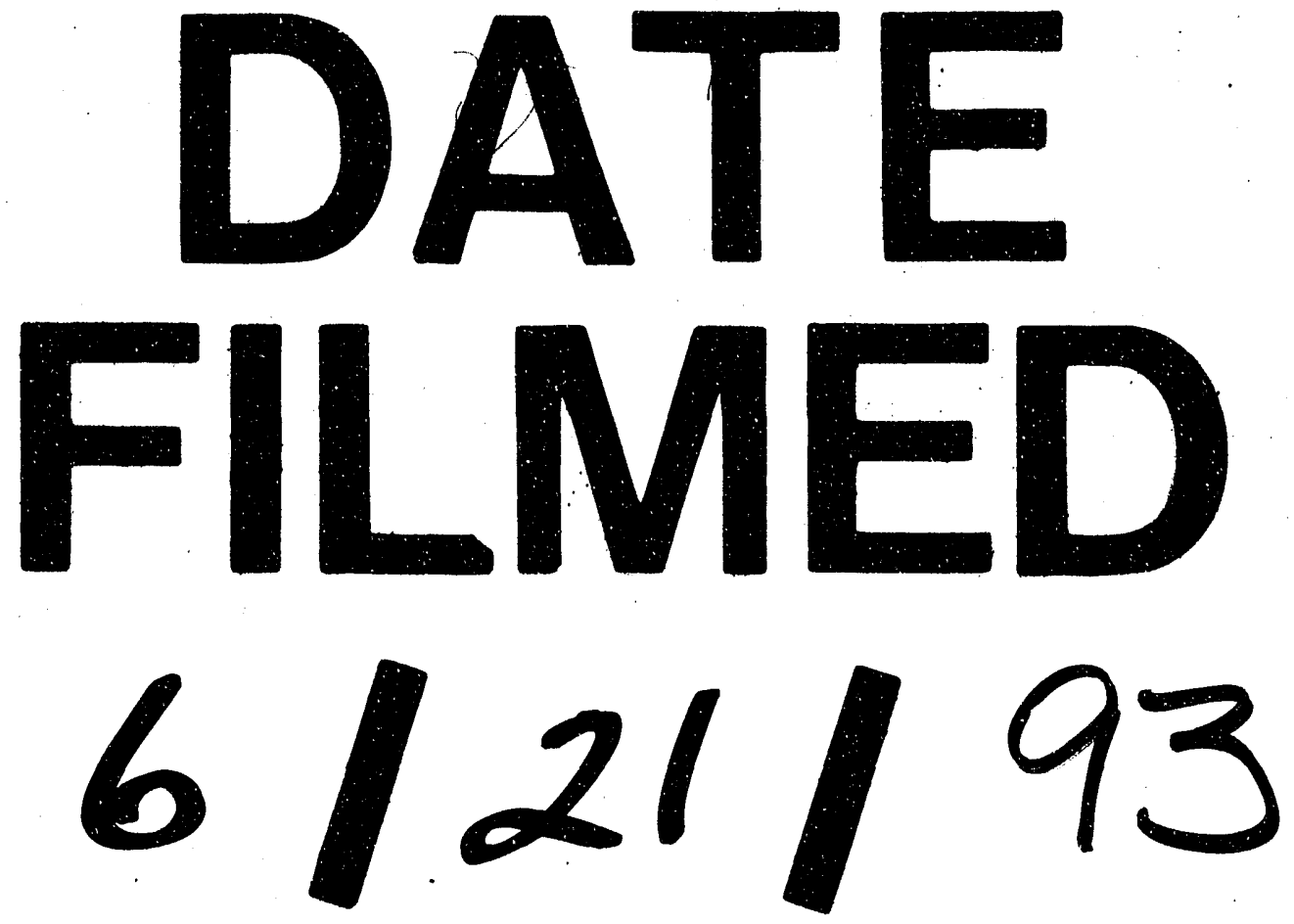
